\title{
An overview of ERP software used in apparel industry
}

\author{
R.Surjit ${ }^{1}$ \\ \{rst.fashion@psgtech.ac.in $\left.{ }^{1}\right\}$ \\ Department of Fashion Technology, PSG College of Technology, Coimbatore, India ${ }^{1}$
}

\begin{abstract}
Enterprise resource planning shortly known as ERP is a business management software that encompasses all the activities and transactions in a company. It helps to get data in real time with seamless interface between all departments. The ERP software is single software that captures the activities of all departments of a company. It has many modules which are nothing but activities performed in each department like sales, procurement, inventory, order planning, etc.
\end{abstract}

Keywords: ERP, Textile, Software, Modules, SAP, Oracle.

\section{Introduction}

Enterprise resource planning (ERP) is a software that is used to manage business. As rightly said by Tadjer, it is a unifying interface of an entire organisation with a single database and single application [1]. The ERP software replaces the traditional MRP, MRPII tools and is a conglomeration of activities of departments put together in a single software. It also replaces the traditional stand alone systems created using FoxPro, COBOL. In ERP, data is entered about various transactions happening in a department of a company and that is obtained as reports for the operational and management teams to look at it and act if needed based on the output. It is one stop solution for getting information from all departments. The different departments that will be having its data in ERP of a textile company are shown in Fig 1.It shows that ERP software encompasses all the department of a company.

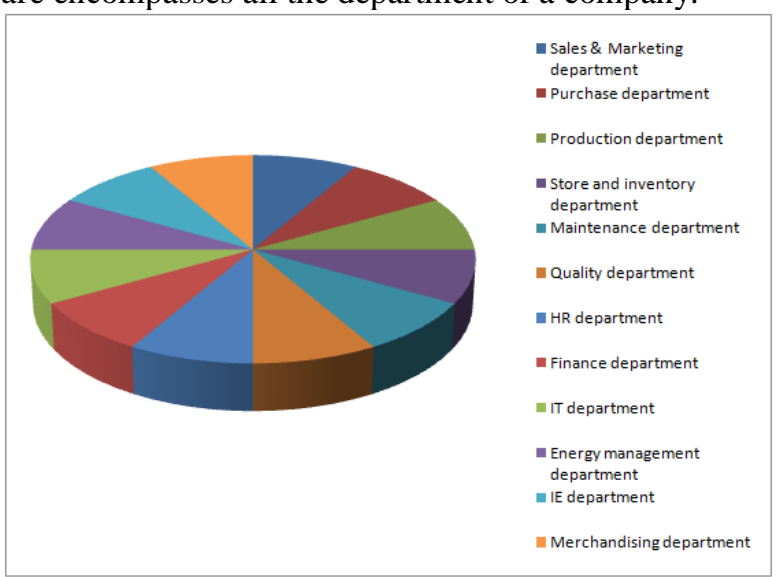

Fig 1. Different departments covered by ERP 
To make it more easily understandable, an example of using ERP software is provided. If a new shirt order is received by merchandising department, they enter it in the system. The same order can be opened by planning department to plan the material, man and machine allocation using the system and a production order can be created online. The created production order can be viewed by purchase department. They will check the availability of material in the store department using ERP software and place an order to the vendor using ERP software. The inventory department will receive the material against the order and enter it in the ERP software. The production department will request the material through ERP software. The material will be issued by the store by putting a store indent through ERP software. Then the production details with respect to machine, name, order number will be entered in the ERP software. The produced material will be quality checked and data of checking will be again entered in the ERP software. The dispatch team will create a dispatch order along with a packing list through ERP software. The material packed according to the packing list will be delivered to the customer along with an invoice prepared by ERP software. The delivery challan and gate pass will also be prepared using the ERP software. The money received for the order based on the invoice sent will be entered in the account receivables section of the ERP software leading to funds realisation. So this example denotes how ERP software is helpful in an apparel company. The entire activity done in an apparel company is entered in the ERP software. It helps each and every stake holder to check the status of the order in the software at appropriate places thereby ensuring a close knit structure of activities avoiding deviation and mistakes.

A question arises on the difference between ERP software and traditional stand alone software. The software that was used before ERP was used for a particular department. Many inventory based software were used. Similarly separate software for payroll like PeopleSoft was used [2]. Software like tally was used for finance related transactions. With the advent of ERP, these stand alone software which were functioning as different islands have all been integrated into a single software. That one software can now provide details between departments to facilitate the process. For example, for paying wages to a worker which is a finance transaction done in separate finance software, the data of the worker, working hours, number of days worked have to be entered in it. If we have an ERP software, then these data can be automatically captured from the HR related database in the same software and used thereby reducing time, efforts and error. The ERP software has many modules in it and is discussed below:

\section{Modules in ERP}

The ERP software consists of various modules. All the modules are integrated as one software. The modules are nothing but the departments of an apparel company grouped based on the activities that are performed in the company. The ERP software manufacturer will have different modules and based on the requirement of the apparel company, the modules can be chosen. The modules are divided into technical and commercial modules [3]. The technical modules and commercial modules are shown in Table 1. 
TABLE I. ERP MODULES [3]

\begin{tabular}{|l|l|}
\hline \multicolumn{2}{|c|}{ MODULES IN ERP } \\
\hline Technical modules & Commercial module \\
\hline Planning module & Sales module \\
\hline Production module & Purchase module \\
\hline Quality module & Inventory module \\
\hline Maintenance module & Human relations module \\
\hline \multirow{5}{*}{} & CRM module \\
\cline { 2 - 2 } & Finance module \\
\cline { 2 - 2 } & Utilities module \\
\cline { 2 - 3 } & Asset management module \\
\cline { 2 - 3 } & Raw material management module \\
\cline { 2 - 2 }
\end{tabular}

These are standard modules available in an ERP software. However many companies have dedicated modules created for garment industry like Time and action module, Industrial engineering module, Merchandising module, Multi style planning module. It varies between ERP manufacturers on what nomenclature is used. These modules mainly indicate the activities done in their respective departments. For example, when inventory module is considered, the transactions done in store department like GRN creation, Stock updation, store indent, issue note, return note, etc. are entered in this module. Similarly, sales related activities are entered in sales module, purchase related activities in purchase module and the list goes on.

\section{Master, transaction and reports}

In each module, there will be master data where the static data like customer information, company information, material information, etc. will be entered. All the one time creation activities for doing a transaction are entered in a master. The transactions like sales order, production order, etc are then created wherein master data is used for it. Based on the transactions done, reports can be generated. The transactions come under dynamic data category. The significance of ERP is the reports that it can generate. From each of these modules, various reports can be obtained. The reports can be customized as per the requirement of the customer. In fact, many ERP software have the option of customers creating their own reports. ERP provides routine, information and analysis reports. The reports can be obtained at various levels. For example, a sales report in a season can be obtained customer based, area based, profit amount based, sales amount based, product based, time based, etc. Similarly hundreds of reports can be generated using ERP which is a major benefit of ERP as reports are obtained within minutes saving time and effort. It leads to better planning of resources.

\section{Benefits of ERP}

The various benefits of ERP are mentioned here. ERP provides operational benefits, managerial benefits, strategic benefits, IT infrastructure benefits and organisational benefits [4]. Staehr et. al. classified the benefits of ERP under environmental context and organizational context [5]. In a study by Gattiker et. al., it was found that ERP leads a company to confidently make customer commitments and to keep them [6]. ERP helps in easy data gathering, provides information at the right time and context. It helps in decision making. It helps in making process corrections. It helps in understanding the requirements in a company. It makes the company and employees more efficient. It leads to quality product output. 
Many companies manufacture this ERP software for textile and apparel. Some companies have standard ERP software which will be suitable for any business including apparel. There are companies which develop ERP only for apparel industry. There are so many players with respect to the companies developing ERP software for apparel industry. In this study, an attempt is made to look at the various apparel ERP software along with their special features so that it will help an apparel company to choose the right ERP for them

\section{Materials And Methods}

This study is carried out by collecting information through google search google scholar, research gate, Elsevier and springer database. An attempt is made to find the various apparel ERP manufacturers from the above sources. Very few research articles have been found with respect to apparel ERP when searched. Different keywords were used related to apparel and ERP to find the relevant software. The majority of information was obtained through Google search. Then the usability of the software, benefits and special features of some of the majorly used ERP software are discussed.

\section{Results And Discussions}

Some of the different apparel ERP software available in the market are SAP business one, Oracle Netsuite ERP, Infor Fashion, FDM 4, Apparel connect, A2000, PolyPM,Align books, Pretture, Expand smERP, Horizon ERP, TYASuite cloud ERP, Focus 9, Axant ERP, Uprow ERP, Sage 300, Ramco ERP [7,8,9,10]. The ERP providers are classified as Tier I, II and III. The big players like Oracle, SAP are classified as Tier I and they cater to large industries with their enhanced functionality. The Tier II vendors are Infor, Epicor, Intex, Datatex, Ramco which serve the medium scale enterprises followed by Tier III providers like Apparel connect, A2000 which provide for small enterprises [3]. The ERP system providers also have different ways of marketing their business. They come as SaaS, PaaS, IaaS. SaaS refers to software as a service where the software is provided and the database management is all done by the ERP provider where the data entered will be encrypted and stored in the database managed by ERP provider at his place called cloud management. In PaaS, platform as a service, the platform including database, operating systems, technology tools are provided by the developer and the apparel company can use that to run multiple software that they have in their company like PLM, CRM, BI, etc. Then comes IaaS where the entire infrastructure including computer, IT technicians are all provided by ERP provider and managed by them.

The cost of the ERP systems varies according to the type of service, functionality, features and usage. In these ERP systems, the major ones which have high market share and the ones suitable for small and medium enterprises with more features are discussed further for their suitability and applicability for increased usage in apparel industries

\section{SAP business one}

SAP SE is a major ERP provider in the world. They provide various software and technology solutions to different verticals. Their ERP software is so versatile and flexible that it can be used for any industry as it has concentrated on the systems. The system remains the same in any company. There is going to be an input, processing and output. So the ERP is 
based on this system which can fit any company. SAP has many ERP software for large, medium and small scale industries. Out of them, SAP business one is a very compact, intelligent ERP software. The strength of SAP is the financial module called as FICO which has great tools for accounting and finance control. It has all the tools inbuilt for accounting, fixed asset management, financial reporting, analysis and controlling [11]. Similarly, it has excellent features like marketing campaign management under sales and customer management, warehousing and integration in purchasing and inventory control module. ABAP programming is used in developing this ERP. It has industry specific functionality and capability and hence it suits apparel industry very well. It has great mobility for access through any devices and helps in decision making across different business verticals at ease. The special features of this software are the business intelligence, analysis and reporting tools possessed by it. It has drill down reports, analytics with clear metrics, dashboards, access to ease in data visualization and real time analytics. Any report can be obtained based on customer requirement. It can be created by the customer thereby making it one of the market leaders in ERP.

\section{Netsuite ERP}

Netsuite ERP of Oracle corporation is a cloud based ERP where the database is maintained by Netsuite. This ERP helps in automating the core processes and increases real time visibility of all activities taking place in a company [12]. It has capability to manage order processing, inventory, purchase, production, warehousing and supply chain with a single application. It helps in making better decisions as it has various tools to capture the operational, transactional and organizational data. Similar to SAP business one, there are various analytical tools available in Netsuite and it provides in depth analysis of the business activity. A global business can be managed by being at a single place with this ERP due to its tremendous interface with all departments. It has tools for optimisation of supply chain, error proof order management and minimize inventory carrying costs. The various modules available are accounting and financial management, global business management, inventory management, order management, supply chain management and warehouse management. The global business management is a very useful module as it helps in doing business globally where the software has provisions to use multiple currencies, adopt to different taxations prevalent in each country and state thereby providing real time access to the right information in the required format. Their warehouse management involves integration with shipping systems leading to easy transition and consolidation of business activities. It has RF device based putaway and picking tasks carried out. It suits to any business based on its universality and hence it fits apparel business also. It can be used for apparel business and production facilities.

\section{FDM4}

FDM4 is a company that specializes in ERP and ECOM. It also has various other solutions like warehouse management solutions which do operate as standalone software. They say that their ERP is based on four tenets which are a. optimize operations, b. provide business agility, c. be customer centric and d. empower employees [13]. They do stand by their tenets as this ERP provides opportunities for thriving a business rather than striving for business. They have apparel ready software, meaning it may not need any customization to use for apparel unlike SAP and Netsuite. The implementation time can be completely reduced. It has financial accounting, purchasing, order processing, inventory, manufacturing, decoration modules. The interesting module here is the decoration module where clients are given more freedom to use the software by having access to uploading art works, designs, provide order quantity changes and various instructions through this module. It gives the 
buyer a free hand to modify requirements as and when required thereby making customer interaction easier. This avoids manual input of data by in house team as the data is entered by the customer during the order creation which is first of its kind in an ERP software. Real time data capturing is possible in this module. It has EASI EDI. EASI refers to embellished activewear standards initiative. If the apparel company is EASI compliant, then they are instantly connected to the biggest network of apparel producers and distributors. It helps the company to be in easy contact with EASI vendors. Their apparel customers include Bella+Canvas, River's end, heritage sportswear, alphabroder, alo, etc.

\section{Infor}

Infor is a company that provides ERP solutions, healthcare activities, hospitality solutions, public sector activities, etc. They have industry ERP which caters to different business verticals. Among them, they have an apparel ERP which is ready for deploying similar to FDM4. They have a software for fashion and apparel industry which can simplify the process and transform the business. They have cloud based ERP which can cater to the changing needs of the fashion and apparel industry. Their special features are that they support models with respect to sustainable circular fashion where there are options available to collaborate with stakeholders to avoid waste, design products for reusing and recycling and increase durability. Their software provides end to end supply chain visibility including einvoicing, multi mode transportation tracking and monitoring. It has options to build visualizations of apparel in $2 \mathrm{D}$ or $3 \mathrm{D}$ models. It leads to increased agility in apparel companies. It provides various tools to increase process performances, reduce costs and improve quality. It has various products like Infor M3, Infor OS, Infor Birst and Infor implementation accelerators [14]. It is mainly concentrating on order management, design management and it has good features with respect to fashion management than complete apparel production.

\section{Apparel connect}

It is an ERP software that is developed exclusively for apparel manufacturers and buying houses. It is a Bangkok based software development company. It is useful for buying houses, OEM manufacturers, CMT/CMPT factories and also for garment and fabric traders [15]. It is a solution that caters to traders also as it has catalogue management, order management, warehousing management, purchasing and financial modules. The CMT and CMPT factories can use it for intelligent planning, production scheduling, multi style planning, line balancing, on time production ensuring, order tracking, vessel tracking, etc. It helps in creating RFQ's (request for quotation) at a very fast pace thereby helping the merchandisers to plan well. It has excellent time and action tools that capture all the phases involved from quotation to production including prototype development. The best part of this ERP is that it can also be used by buying houses for multi option sourcing, multi dimensional costing thereby leading to options which help in facing day to day changes. The various modules in apparel connect are development which involves pre order booking processes, order book, line planning, workflow, stores, cut room, BOM (bill of materials), Work in progress. There are optional modules like fabric processing, QC inspections and client management.

\section{Polygon software}

This software from Polygon is a combination of ERP and PLM for apparel industry. They also provide CAD solutions for apparel industry worldwide. They have all the required modules for an effective apparel ERP. The software includes production planning and cut planning. It can be integrated with CAD software [16]. Web browser and client server technology is used by them which replace the desktop based applications. It integrates PLM and ERP thereby leading to reduced inventory costs, reducing errors, reducing lead times and 
improving the production scheduling and execution process. It helps in style development using PLM techniques. The production requirements can be planned based on the sales cycle and buying trends analysis. It also interfaces with customer better as it provides accurate and real time responses to the customers. It has complete order tracking tools and cost estimation tools.

A2000

A2000 ERP is rated as the highest customer satisfaction provider by Apparel magazine. This software helps designers, manufacturers, wholesalers and retailers. It is a complete end to end solution. It concentrates on limiting human work and thereby reducing errors cost by that. It has B2B, B2C connect modules which help in seamlessly connecting with upstream and downstream supply chain entities respectively. It makes the interaction between all the stakeholders easy and in real time. The various other modules available are SCM, MRP, warehouse management, EDI, vendor portal and finance. Their mantra is to avoid rework and make the system flexible and reliable.

The above discussion on the different ERP software for apparel gives a clear idea on the type of providers available in the market along with their benefits and features. The apparel company has to spend considerable time in choosing the right vendor as the entire company activities will be done in ERP once implemented thereby replacing the legacy systems.

\section{Conclusion}

This study concentrated on analysing the various apparel ERP software available in the market. The concept of ERP, its modules and its benefits are discussed. The importance of reports that can be generated with ERP is discussed. The ERP software that can be used for apparel is detailed. The special features, benefits and modules available in different ERP software are discussed. It is found that SAP business one, Oracle Netsuite are ERP software that can be used for big apparel companies and they have to be customized to the requirements of the apparel company. However there are other ERP software which are apparel ready like FDM4, Infor which can be installed quickly but may not have all the features of a SAP or Netsuite ERP. They do have various tools which aid in improved productivity management of an apparel company. There are also apparel specific ERP products produced by Apparel connect, Polygon software and A2000 which include all the activities done in an apparel company. They are also found to have many features that can help apparel manufacturers, buying houses and traders. It can be concluded that there are many players available to implement ERP in an apparel company at ease so that companies can benefit from the tools available in the software.

\section{References}

[1] Tadjer, R., 1998. Enterprise resource planning. Internetweek, pp.40-44.

[2] Sumner, M., 2000. Risk factors in enterprise-wide/ERP projects. Journal of information technology, 15(4), pp.317-327.

[3] Surjit, R., Rathinamoorthy, R. and Vardhini, K.V., 2016. ERP for textiles and apparel industry. CRC Press.

[4] Shang, S. and Seddon, P.B., 2000. A comprehensive framework for classifying the benefits of ERP systems. AMCIS 2000 proceedings, p.39.

[5] Staehr, L., Shanks, G. and Seddon, P.B., 2012. An explanatory framework for achieving business benefits from ERP systems. Journal of the Association for Information Systems, 13(6), p.2. 
[6] Gattiker, T.F. and Goodhue, D.L., 2004. Understanding the local-level costs and benefits of ERP through organizational information processing theory. Information \& management, 41(4), pp.431443.

[7] Carlton, R. 2019. Five top apparel ERP systems for your software shortlist, https://www.erpfocus.com/top-apparel-erp-systems.html. Accessed on 20 September 2021.

[8] Anonymous, n.d. Apparel ERP software, https://www.softwareadvice.com/manufacturing/apparelmanufacturing-software-comparison/. Accessed on 20 September 2021.

[9] Anonymous, n.d. Best ERP software for apparel business, https://www.softwaresuggest.com/erpsoftware/apparel-industry. Accessed on 20 September 2021

[10] A2000, https://www.a2000software.com/. Accessed on 15 September 2021

[11] SAP business one, https://accelontech.com/erp/sap-business-one/. Accessed on 15 September 2021

[12] Netsuite ERP, https://www.netsuite.com/portal/products/erp.shtml. Accessed on 10 September 2021

[13] FDM4, https://www.fdm4.com/. Accessed on 15 September 2021

[14] Infor, https://www.infor.com/industries/fashion. Accessed on 15 September 2021

[15] Apparel connect, http://www.apparel-connect.com/. Accessed on 15 September 2021

[16] Polygon software, https://polypm.com/. Accessed on 15 September 2021 